\title{
SOME NEW INEQUALITIES BETWEEN IMPORTANT MEANS AND APPLICATIONS TO KY FAN - TYPE INEQUALITIES
}

\author{
JAMAL ROOIN AND MEHDI HASSANI
}

Abstract. In this paper, mainly using the convexity of the function $\frac{a^{x}-b^{x}}{c^{x}-d^{x}}$ and convexity or concavity of the function $\ln \frac{a^{x}-b^{x}}{c^{x}-d^{x}}$ on the real line, where $a>b \geqslant c>d>0$ are fixed real numbers, we obtain some important relations between various important means of these numbers. Also, we apply the obtained results to Ky Fan type inequalities and get some new refinements.

Mathematics subject classification (2000): 26D15, 26A06, 39B62.

Key words and phrases: convexity, Jensen inequality, means, Ky Fan's inequality.

\section{REFERENCES}

[1] H. AlzER, The inequality of Ky Fan and related results, Acta Appl. Math., 38, (1995), 305-354.

[2] H. AlzER, Ungleichungen für geometrische und arithmetische Mittelwerte, Proc. Kon. Nederl. Akad. Wetensch., 91, (1988), 365-374.

[3] E. F. Beckenbach, R. Bellman, Inequalities, Springer-Verlag, Berlin, 1961.

[4] D. BorweIn, J. BorweIn AND J. RooIn, Problem 11009, Amer. Math. Monthly, 110, (4) (2003), 341.

[5] P. S. Bullen, D. S. Mitrinović AND P. M. Vasić, Means and their inequalities, Reidel, Dordrecht, 1988.

[6] A. Dinghas, Some identities between arithmetic means and the other elementary symmetric functions of $n$ numbers, Math. Ann., 120, (1948), 154-157.

[7] E. B. LeACh, M. C. Sholander, Extended mean values, Amer. Math. Monthly, 85, (1978), 84-90.

[8] E. B. LEACH, M. C. ShOlander, Extended mean values II, J. Math. Anal. Appl., 92, (1983), 207-223.

[9] E. B. LeACH, M. C. Sholander, Multi-variable extended mean values, J. Math. Anal. Appl., 104, (1984), 390-407.

[10] J. E. PEČARIĆ, H. Alzer, On Ky Fan's inequality, Math. Panonica, 6, (1995), 85-93.

[11] J. Rooln, On Ky Fan's Inequality and its Additive Analogues, Math. Inequal. Appl., 6, (4) (2003), 595-604. 